\title{
Symbolic Computation for Moments and Filter Coefficients of Scaling Functions
}

\author{
Georg Regensburger ${ }^{1}$ and Otmar Scherzer ${ }^{2}$ \\ Institute of Mathematics ${ }^{1}$, \\ Department of Computer Science ${ }^{2}$, \\ University Innsbruck, \\ Techniker Str. 25, \\ A-6020 Innsbruck, \\ Austria. \\ E-mail: \{georg.regensburger, otmar.scherzer\}@uibk.ac.at
}

December 29, 2005

AMS Subject Classification: 42C40, 65T60, 13P10, 94A12, 05A10, 33C45

\begin{abstract}
Algebraic relations between discrete and continuous moments of scaling functions are investigated based on the construction of Bell polynomials. We introduce families of scaling functions which are parametrized by moments. Filter coefficients of scaling functions and wavelets are computed with computer algebra methods (in particular Gröbner bases) using relations between moments. Moreover, we propose a novel concept for data compression based on parametrized wavelets.
\end{abstract}

Keywords: scaling functions, moments, Bell polynomials, wavelets, Gröbner bases, data compression

Suggested running title: Moments and Filter Coefficients of Scaling Functions

\section{Introduction}

Discrete (real or complex) filter coefficients $\left\{h_{k}: k=0, \ldots, N\right\}$ in the dilation equation of a scaling function

$$
\phi(x)=\sum_{k=0}^{N} h_{k} \phi(2 x-k),
$$

are used in many areas of applications, such as for instance data compression; scaling functions (and in turn filter coefficients) are the basis for constructing wavelets (see e.g. Daubechies [12,13], Mallat [20], Strang \& Nguyen [27]) and also play a fundamental role in subdivision schemes (see e.g. Cavaretta et al. [7] and Rioul [22]). 
Filter coefficients are determined by the continuous moments

$$
M_{n}=\int x^{n} \phi(x) d x
$$

and discrete moments

$$
m_{n}=\sum_{k=0}^{N} h_{k} k^{n}
$$

respectively.

In Section 2 we study algebraic relations between discrete and continuous moments - in contrast to the literature where recursive relations have been established (see e.g. Strang \& Nguyen [27]). In particular we express the $n$th continuous moment as a polynomial of the first $n$ discrete moments and vice versa. The polynomials are related to Bell polynomials. The definition of Bell polynomials as well as some of their elementary properties are stated in the Appendix.

We recall and extend polynomial relations between moments of scaling functions associated with orthogonal wavelets in Section 3. In wavelet theory smoothness and the approximation order of scaling functions are related to vanishing moments conditions for wavelets (see Daubechies [12, 13], Strang \& Nguyen [27] or Unser \& Blu [29] for a recent survey). The study of parametrized scaling functions and wavelets is along the lines of Daubechies [14] who showed that more symmetry, better frequency resolution as well as regularity of scaling functions associated with wavelets can be achieved by using additional degrees of freedom obtained by giving up some higher order vanishing moment conditions in the constitutive equations. We compute analytical expressions of parametrized scaling function families using symbolic computation methods (in particular Gröber bases) (cf. Section 3.2). In our work we use parametrization with respect to the discrete and continuous moments. In Subsection 3.3 we propose a novel concept of data compression using parametrized scaling functions and wavelets. For compression, the coefficients of the wavelet expansion of the data are computed for a series of parameters. The parameter yielding optimal compression rates is selected. The compressed data consists of the coefficients and the single parameter. These data are sufficient for decoding. A numerical example illustrating the compression idea is presented.

\section{Continuous and Discrete Moments}

We recall a well-known recursive relation between discrete and continuous moments (see for example Strang \& Nguyen [27, p. 396]).

Lemma 1. Let $\phi$ be a scaling function satisfying $M_{0}=\int \phi=1$. Then $m_{0}=2$ and

$$
\begin{aligned}
M_{n} & =\frac{1}{2^{n+1}-2} \sum_{i=1}^{n}\left(\begin{array}{c}
n \\
i
\end{array}\right) m_{i} M_{n-i}, \\
m_{n} & =\left(2^{n+1}-2\right) M_{n}-\sum_{i=1}^{n-1}\left(\begin{array}{c}
n \\
i
\end{array}\right) m_{i} M_{n-i}, \quad \text { for } n=1,2 \ldots
\end{aligned}
$$


In particular, for $n=1, \ldots, 4$, subsequent application of (2.2) shows that

$$
\begin{aligned}
& M_{1}=\frac{1}{2} m_{1} \\
& M_{2}=\frac{1}{6} m_{1}^{2}+\frac{1}{6} m_{2} \\
& M_{3}=\frac{1}{28} m_{1}^{3}+\frac{1}{7} m_{1} m_{2}+\frac{1}{14} m_{3} \\
& M_{4}=\frac{1}{210} m_{1}^{4}+\frac{11}{210} m_{1}^{2} m_{2}+\frac{8}{105} m_{1} m_{3}+\frac{1}{30} m_{2}^{2}+\frac{1}{30} m_{4}
\end{aligned}
$$

and

$$
\begin{aligned}
& m_{1}=2 M_{1} \\
& m_{2}=-4 M_{1}^{2}+6 M_{2} \\
& m_{3}=12 M_{1}^{3}-24 M_{1} M_{2}+14 M_{3} \\
& m_{4}=-48 M_{1}^{4}+120 M_{1}^{2} M_{2}-64 M_{1} M_{3}-36 M_{2}^{2}+30 M_{4} .
\end{aligned}
$$

These examples indicate that the continuous moments can be expressed as polynomials with rational coefficients in the discrete moment variables. The discrete moments are polynomials with integer coefficients in the continuous moments variables. In this section we derive the algebraic structure of these polynomials. To this end we set $p_{0}=1$ and define recursively the polynomials

$$
p_{n}:=p_{n}\left(x_{1}, \ldots, x_{n}\right):=\frac{1}{2^{n+1}-2} \sum_{i=1}^{n}\left(\begin{array}{l}
n \\
i
\end{array}\right) x_{i} p_{n-i} \in \mathbb{Q}\left[x_{1}, \ldots, x_{n}\right],
$$

and

$$
q_{n}:=q_{n}\left(x_{1}, \ldots, x_{n}\right)=\left(2^{n+1}-2\right) x_{n}-\sum_{i=1}^{n-1}\left(\begin{array}{l}
n \\
i
\end{array}\right) x_{n-i} q_{i} \in \mathbb{Z}\left[x_{1}, \ldots, x_{n}\right] .
$$

By induction it can easily be shown that $p_{n}$ and $q_{n}$ are weighted homogeneous of degree $n$ with $\operatorname{deg} x_{i}=i{ }^{*}$ From Lemma 1 we see that

$$
M_{n}=p_{n}\left(m_{1}, \ldots, m_{n}\right) \text { and } m_{n}=q_{n}\left(M_{1}, \ldots, M_{n}\right) .
$$

In the following two subsections we analyze the polynomials $p_{n}$ and $q_{n}$. We solve the recurrence equations (2.3) and (2.4) by giving explicit formulas for the polynomials. Apart from the theoretical interest these formulas allow us to calculate $n$th polynomial without knowing the previous ones.

\subsection{Continuous $\Rightarrow$ Discrete Moments}

We derive formulas for the polynomials $q_{n}$, defined in (2.4). For this purpose we use linear combinations of partial Bell polynomials $B_{n, k}$ (see Definition 12). We define

$$
Q_{n, k}=(-1)^{k} k ! B_{n, k}, \quad \text { for } n, k \in \mathbb{N} .
$$

\footnotetext{
${ }^{*}$ Let $d_{i}, i=1, \ldots, n$, be positive integers. The weighted degree of a monomial $x^{\alpha}=x_{1}^{\alpha_{1}} \cdots x_{n}^{\alpha_{n}}$ is $\sum_{i=1}^{n} \alpha_{i} d_{i}$. We refer to $d_{i}$ as the weight (or degree) of $x_{i}$ and write deg $x_{i}=d_{i}$. A polynomial is called weighted homogeneous if all of its monomials have the same weighted degree.
} 
From (4.30) in the Appendix we see that

$$
\begin{aligned}
Q_{n, k} & :=Q_{n, k}\left(x_{1}, \ldots, x_{n-k+1}\right) \\
& =\sum_{\substack{i_{1}+\ldots+i_{k}=n \\
i_{j}>0}}(-1)^{k}\left(\begin{array}{c}
n \\
i_{1}, \ldots, i_{k}
\end{array}\right) x_{i_{1}} \cdots x_{i_{k}} \in \mathbb{Z}\left[x_{1}, \ldots, x_{n-k+1}\right],
\end{aligned}
$$

where

$$
\left(\begin{array}{c}
n \\
i_{1}, \ldots, i_{k}
\end{array}\right)=\frac{n !}{i_{1} ! \ldots i_{k} !}
$$

Note that

$$
\left(\begin{array}{c}
n \\
i_{1}, \ldots, i_{k}
\end{array}\right)=\left(\begin{array}{c}
n \\
i_{1}
\end{array}\right)\left(\begin{array}{c}
n-i_{1} \\
i_{2}, \ldots, i_{k}
\end{array}\right)
$$

We define

$$
Q_{0,0}=1, \quad Q_{n, 0}=Q_{0, k}=0, \quad \text { for } n, k \in \mathbb{N},
$$

and

$$
Q_{n}:=Q_{n}\left(x_{1}, \ldots, x_{n}\right)=\sum_{k=0}^{n} Q_{n, k} \in \mathbb{Z}\left[x_{1}, \ldots, x_{n}\right], \quad \text { for } n \in \mathbb{N}_{0} .
$$

The first polynomials $Q_{n}$ are:

$$
\begin{aligned}
& Q_{0}=1 \\
& Q_{1}=-x_{1} \\
& Q_{2}=2 x_{1}^{2}-x_{2} \\
& Q_{3}=-6 x_{1}^{3}+6 x_{1} x_{2}-x_{3} \\
& Q_{4}=24 x_{1}^{4}-36 x_{1}^{2} x_{2}+6 x_{2}^{2}+8 x_{1} x_{3}-x_{4} .
\end{aligned}
$$

The polynomials $Q_{n}$ fit in the class of potential polynomials (see Comtet [10, p. 141]). The following lemma provides recurrence relations for the polynomials $Q_{n, k}$ and $Q_{n}$.

Lemma 2. Let $n \in \mathbb{N}$. The polynomials $Q_{n, k}$ and $Q_{n}$ satisfy

(1) For $1 \leq k \leq n$

$$
Q_{n, k}=-\sum_{i=1}^{n}\left(\begin{array}{l}
n \\
i
\end{array}\right) x_{i} Q_{n-i, k-1}
$$

(2)

$$
Q_{n}=-\sum_{i=1}^{n}\left(\begin{array}{l}
n \\
i
\end{array}\right) x_{i} Q_{n-i}=-\sum_{i=0}^{n-1}\left(\begin{array}{l}
n \\
i
\end{array}\right) x_{n-i} Q_{i} .
$$

Proof. Let $n \in \mathbb{N}$ and $2 \leq k \leq n$. From (2.5) and (2.6) it follows that

$$
Q_{n, k}=-\sum_{i=1}^{n-1}\left(\begin{array}{l}
n \\
i
\end{array}\right) x_{i} Q_{n-i, k-1} .
$$

This together with $Q_{0, k-1}=0$ gives the first assertion for $2 \leq k \leq n$. 
Let $k=1$. Since $Q_{0,0}=1$ and $Q_{n-i, 0}=0$ for $i=1, \ldots, n-1$ it follows that

$$
Q_{n, 1}=-x_{n}=-\sum_{i=1}^{n}\left(\begin{array}{c}
n \\
i
\end{array}\right) x_{i} Q_{n-i, 0} .
$$

Moreover, since $Q_{n, 0}=0$, we have

$$
Q_{n}=\sum_{k=0}^{n} Q_{n, k}=\sum_{k=1}^{n} Q_{n, k}
$$

Using (2.8) this implies that

$$
Q_{n}=-\sum_{k=1}^{n} \sum_{i=1}^{n}\left(\begin{array}{c}
n \\
i
\end{array}\right) x_{i} Q_{n-i, k-1}=-\sum_{i=1}^{n}\left(\begin{array}{c}
n \\
i
\end{array}\right) x_{i} \sum_{k=1}^{n} Q_{n-i, k-1}
$$

Since $Q_{n, k}=0$ for $k>n$, we have $Q_{n-i, k-1}=0$ for $k-1>n-i$. Therefore

$$
\sum_{k=1}^{n} Q_{n-i, k-1}=\sum_{k=1}^{n-i+1} Q_{n-i, k-1}=Q_{n-i}
$$

The second assertion follows from the last two equations.

The following theorem gives an explicit formula for the polynomials $q_{n}$ in terms of the known polynomials $Q_{n}$.

Theorem 3. For $n \in \mathbb{N}$

$$
q_{n}=\sum_{i=1}^{n}\left(2^{i+1}-2\right)\left(\begin{array}{c}
n \\
i
\end{array}\right) x_{i} Q_{n-i} .
$$

Proof. For $n=1$ the assertion is true since $q_{1}=2 x_{1}$ by (2.4) and

$$
2\left(\begin{array}{l}
1 \\
1
\end{array}\right) x_{1} Q_{0}=2 x_{1}
$$

Let

$$
\tilde{q}_{n}=\sum_{i=1}^{n}\left(2^{i+1}-2\right)\left(\begin{array}{c}
n \\
i
\end{array}\right) x_{i} Q_{n-i}, \quad n=1,2, \ldots .
$$

We proof that $q_{n}$ and $\tilde{q}_{n}$ both satisfy the recurrence (2.4), which then implies that they are identical. To this end we show that

$$
\begin{aligned}
\tilde{q}_{n} & =\left(2^{n+1}-2\right) x_{n}+\sum_{i=1}^{n-1}\left(2^{i+1}-2\right)\left(\begin{array}{c}
n \\
i
\end{array}\right) x_{i} Q_{n-i} \\
& =\left(2^{n+1}-2\right) x_{n}-\sum_{i=1}^{n-1}\left(\begin{array}{c}
n \\
i
\end{array}\right) \tilde{q}_{i} x_{n-i}, \quad \text { for } n \in \mathbb{N} .
\end{aligned}
$$


From (2.10) it follows that

$$
-\sum_{i=1}^{n-1}\left(\begin{array}{l}
n \\
i
\end{array}\right) \tilde{q}_{i} x_{n-i}=-\sum_{i=1}^{n-1}\left(\begin{array}{c}
n \\
i
\end{array}\right)\left(\sum_{j=1}^{i}\left(2^{j+1}-2\right)\left(\begin{array}{l}
i \\
j
\end{array}\right) x_{j} Q_{i-j}\right) x_{n-i} .
$$

Using the binomial identity

$$
\left(\begin{array}{l}
n \\
i
\end{array}\right)\left(\begin{array}{l}
i \\
j
\end{array}\right)=\left(\begin{array}{l}
n \\
j
\end{array}\right)\left(\begin{array}{c}
n-j \\
i-j
\end{array}\right)
$$

and interchanging the order of summation gives

$$
-\sum_{i=1}^{n-1}\left(\begin{array}{l}
n \\
i
\end{array}\right) \tilde{q}_{i} x_{n-i}=-\sum_{j=1}^{n-1}\left(\begin{array}{l}
n \\
j
\end{array}\right)\left(2^{j+1}-2\right) x_{j}\left(\sum_{i=j}^{n-1}\left(\begin{array}{c}
n-j \\
i-j
\end{array}\right) Q_{i-j} x_{n-i}\right) .
$$

From (2.9) it follows that

$$
\sum_{i=j}^{n-1}\left(\begin{array}{c}
n-j \\
i-j
\end{array}\right) Q_{i-j} x_{n-i}=\sum_{i=0}^{n-1-j}\left(\begin{array}{c}
n-j \\
i
\end{array}\right) Q_{i} x_{n-j-i}=-Q_{n-j}
$$

Using this identity in (2.13) yields

$$
-\sum_{i=1}^{n-1}\left(\begin{array}{c}
n \\
i
\end{array}\right) \tilde{q}_{i} x_{n-i}=\sum_{i=1}^{n-1}\left(\begin{array}{c}
n \\
i
\end{array}\right)\left(2^{i+1}-2\right) x_{i} Q_{n-i}
$$

and the assertion (2.11) is proved.

The first polynomials $q_{n}$ are:

$$
\begin{aligned}
q_{1}= & 2 x_{1} \\
q_{2}= & -4 x_{1}^{2}+6 x_{2} \\
q_{3}= & 12 x_{1}^{3}-24 x_{1} x_{2}+14 x_{3} \\
q_{4}= & -48 x_{1}^{4}+120 x_{1}{ }^{2} x_{2}-64 x_{1} x_{3}-36 x_{2}^{2}+30 x_{4} \\
q_{5}= & 240 x_{1}^{5}-720 x_{1}{ }^{3} x_{2}+360 x_{1}{ }^{2} x_{3}+420 x_{1} x_{2}^{2}-160 x_{1} x_{4}-200 x_{2} x_{3}+62 x_{5} \\
q_{6}= & -1440 x_{1}^{6}+5040 x_{1}^{4} x_{2}-2400 x_{1}^{3} x_{3}-4320 x_{1}^{2} x_{2}^{2}+1020 x_{1}^{2} x_{4}+2640 x_{1} x_{2} x_{3} \\
& +540 x_{2}^{3}-384 x_{1} x_{5}-540 x_{2} x_{4}-280 x_{3}^{2}+126 x_{6} .
\end{aligned}
$$

\subsection{Discrete $\Rightarrow$ Continuous Moments}

In this section we further analyze the polynomials $p_{n}$, defined in (2.3). We give explicit formulas for the polynomials as a sum over compositions (for a definition of compositions we refer to Definition 13). 
Let $k, n \in \mathbb{N}$. We define

$$
\begin{aligned}
p_{n, k} & :=p_{n, k}\left(x_{1}, \ldots, x_{n}\right) \\
& =\sum_{\substack{i_{1}+\cdots+i_{k}=n \\
i_{j}>0}} c_{i_{1} \cdots i_{k}}^{n}\left(\begin{array}{c}
n \\
i_{1}, \ldots, i_{k}
\end{array}\right) x_{i_{1}} \cdots x_{i_{k}} \in \mathbb{Q}\left[x_{1}, \ldots, x_{n-k+1}\right],
\end{aligned}
$$

with

$$
c_{i_{1} \cdots i_{k}}^{n}=\frac{1}{\left(2^{n+1}-2\right)\left(2^{n+1-i_{1}}-2\right) \cdots\left(2^{n+1-i_{1}-\cdots-i_{k-1}}-2\right)} .
$$

We define $p_{0,0}=1$ and $p_{n, 0}=p_{0, k}=0$.

We note that

$$
p_{n, k}=0, \quad \text { for } k>n .
$$

The sum (2.14) is over all compositions of $n$ in $k$ parts. We recall the analogy with the constitutive equations for the partial Bell polynomials (cf. (4.30)). However, here in contrast to Bell polynomials, the coefficients $c_{i_{1} \ldots i_{k}}^{n}$ depend on the particular order of the numbers $i_{1}, \ldots, i_{k}$.

In the following theorem we establish recurrence relations for the polynomials $p_{n, k}$ and give a formula for $p_{n}$.

Theorem 4. The polynomials $p_{n, k}$ and $p_{n}$ satisfy

(1) For $n \in \mathbb{N}$ and $1 \leq k \leq n$

$$
p_{n, k}=\frac{1}{\left(2^{n+1}-2\right)} \sum_{i=1}^{n}\left(\begin{array}{l}
n \\
i
\end{array}\right) x_{i} p_{n-i, k-1} .
$$

(2) For $n \in \mathbb{N}_{0}$

$$
p_{n}=\sum_{k=0}^{n} p_{n, k}
$$

Proof. Let $n \in \mathbb{N}$ and $2 \leq k \leq n$. The relation

$$
c_{i_{1} \ldots i_{k}}^{n}=\frac{1}{\left(2^{n+1}-2\right)} c_{i_{2} \ldots i_{k}}^{n-i_{1}}
$$

and the binomial identity (2.6) with (2.14) show that

$$
p_{n, k}=\frac{1}{\left(2^{n+1}-2\right)} \sum_{i=1}^{n-1}\left(\begin{array}{l}
n \\
i
\end{array}\right) x_{i} p_{n-i, k-1} .
$$

This together with $p_{0, k-1}=0$ gives the first assertion for $2 \leq k \leq n$. For $k=1$ it follows from $p_{0,0}=1$ and $p_{n, 0}=0$ that

$$
p_{n, 1}=\frac{1}{\left(2^{n+1}-2\right)} x_{n}=\frac{1}{\left(2^{n+1}-2\right)} \sum_{i=1}^{n}\left(\begin{array}{l}
n \\
i
\end{array}\right) x_{i} p_{n-i, 0} .
$$


For the second claim we define

$$
\tilde{p}_{n}=\sum_{k=0}^{n} p_{n, k}, \quad n=0,1, \ldots
$$

Since $p_{0}=\tilde{p}_{0}=1$, it is sufficient to prove that $\tilde{p}_{n}$ and $p_{n}$ both satisfy the recurrence equation (2.3), that is, it suffices to show that

$$
\tilde{p}_{n}=\frac{1}{2^{n+1}-2} \sum_{i=1}^{n}\left(\begin{array}{l}
n \\
i
\end{array}\right) x_{i} \tilde{p}_{n-i}, \quad \text { for } n \in \mathbb{N} .
$$

Since $p_{n, 0}=0$ it follows from (2.16) that

$$
\tilde{p}_{n}=\sum_{k=1}^{n} p_{n, k}=\frac{1}{\left(2^{n+1}-2\right)} \sum_{i=1}^{n}\left(\begin{array}{c}
n \\
i
\end{array}\right) x_{i} \sum_{k=1}^{n} p_{n-i, k-1} .
$$

From (2.15) it follows that

$$
\sum_{k=1}^{n} p_{n-i, k-1}=\sum_{k=1}^{n-i+1} p_{n-i, k-1}=\sum_{k=0}^{n-i} p_{n-i, k}=\tilde{p}_{n-i} .
$$

This shows the assertion.

The first polynomials $p_{n}$ are:

$$
\begin{aligned}
& p_{0}=1 \\
& p_{1}=\frac{1}{2} x_{1} \\
& p_{2}=\frac{1}{6} x_{1}^{2}+\frac{1}{6} x_{2} \\
& p_{3}=\frac{1}{28} x_{1}^{3}+\frac{1}{7} x_{1} x_{2}+\frac{1}{14} x_{3} \\
& p_{4}=\frac{1}{210} x_{1}^{4}+\frac{11}{210} x_{1}^{2} x_{2}+\frac{8}{105} x_{1} x_{3}+\frac{1}{30} x_{2}^{2}+\frac{1}{30} x_{4} \\
& p_{5}=\frac{1}{2604} x_{1}^{5}+\frac{13}{1302} x_{1}^{3} x_{2}+\frac{43}{1302} x_{1}^{2} x_{3}+\frac{67}{2604} x_{1} x_{2}^{2}+\frac{4}{93} x_{1} x_{4}+\frac{25}{651} x_{2} x_{3}+\frac{1}{62} x_{5} .
\end{aligned}
$$

Daubechies \& Lagarias $[15,16]$ consider scaling functions satisfying a dilation equation of the form

$$
\phi(x)=\sum_{k=0}^{N} h_{k} \phi\left(\alpha x-\beta_{k}\right),
$$

with real numbers $\alpha>1$ and $\beta_{0}<\beta_{1}<\cdots<\beta_{N}$. The results stated so far can easily be modified to this more general situation.

We define the $n$th discrete moment by

$$
m_{n}=\sum_{k=0}^{N} h_{k} \beta_{k}^{n}
$$


Again we assume that $M_{0}=\int \varphi=1$. Then (2.17) implies $m_{0}=\alpha$ and

$$
\begin{aligned}
& M_{n}=\frac{1}{\alpha^{n+1}-\alpha} \sum_{i=1}^{n}\left(\begin{array}{c}
n \\
i
\end{array}\right) m_{i} M_{n-i}, \\
& m_{n}=\left(\alpha^{n+1}-\alpha\right) M_{n}-\sum_{i=1}^{n-1}\left(\begin{array}{c}
n \\
i
\end{array}\right) m_{i} M_{n-i}, \quad \text { for } n=1,2, \ldots
\end{aligned}
$$

In this case the polynomials $p_{n}$ and $q_{n}$ are defined recursively by $p_{0}^{\alpha}=1$ and

$$
\begin{aligned}
& p_{n}^{\alpha}=\frac{1}{\alpha^{n+1}-\alpha} \sum_{i=1}^{n}\left(\begin{array}{c}
n \\
i
\end{array}\right) x_{i} p_{n-i}^{\alpha}, \\
& q_{n}^{\alpha}=\left(\alpha^{n+1}-\alpha\right) x_{n}-\sum_{i=1}^{n-1}\left(\begin{array}{c}
n \\
i
\end{array}\right) x_{n-i} q_{i}^{\alpha} .
\end{aligned}
$$

Replacing the dilation factor 2 by $\alpha$ in the proof of Theorem 3 we see that

$$
q_{n}^{\alpha}=\sum_{i=1}^{n}\left(\alpha^{i+1}-\alpha\right)\left(\begin{array}{c}
n \\
i
\end{array}\right) x_{i} Q_{n-i}, \quad \text { for } n \in \mathbb{N},
$$

with $Q_{n}$ defined as in (2.7). The generalization of Theorem 4 reads as follows

$$
p_{n}^{\alpha}=\sum_{k=0}^{n} p_{n, k}^{\alpha}, \quad \text { for } n \in \mathbb{N}_{0}
$$

where

$$
p_{n, k}^{\alpha}=\sum_{\substack{i_{1}+\cdots+i_{k}=n \\
i_{j}>0}} c_{i_{1} \cdots i_{k}}^{n}\left(\begin{array}{c}
n \\
i_{1}, \ldots, i_{k}
\end{array}\right) x_{i_{1}} \cdots x_{i_{k}} \in \mathbb{Q}\left[x_{1}, \ldots, x_{n-k+1}\right]
$$

and

$$
c_{i_{1} \ldots i_{k}}^{n}=\frac{1}{\left(\alpha^{n+1}-\alpha\right)\left(\alpha^{n+1-i_{1}}-\alpha\right) \cdots\left(\alpha^{n+1-i_{1}-\cdots-i_{k-1}}-\alpha\right)} .
$$

\section{Moments and Filter Coefficients}

The goal of this section is twofold. In the first subsection we recall and extend polynomial relations between moments of scaling functions associated with orthonormal wavelets. The second subsection is devoted to a study of parametrized wavelets. We give an application of parametrized wavelets to compression in the last subsection.

Daubechies [14] showed that more symmetry, better frequency solution and better regularity for scaling functions associated with wavelets can be obtained with the same number of filter coefficients, by neglecting some higher order vanishing moment conditions. She calculated a parametrized family of wavelets with four filter coefficients [12]. In the case of more than four filter coefficients, she replaced various vanishing moment conditions for the associated wavelet and computed solutions of the resulting system for the filter coefficients numerically. In our work we introduce moments of the scaling 
function as parameters and give up one vanishing moment condition. The resulting algebraic system for the filter coefficients is solved with symbolic methods, in particular by using Gröbner bases. Our approach differs essentially from other work on symbolic computation of wavelets coefficients which aim to calculate a finite number of solutions. Here we calculate infinitely many solutions, which are due to the additional degree of freedom imposed by neglecting a vanishing moment condition. Applications of Gröbner bases to the design of wavelets and digital filters are for example described in Chyzak et al. [8], Lebrun \& Selesnick [18], Lebrun \& Vetterli [19] and Selesnick \& Burrus [25]. Gröbner bases were introduced by Buchberger in [4] and [5]. For an introduction see for example Cox et al. [11]. In Subsection 3.3 we use the parametrized scaling functions and wavelets for data compression. The idea is to find the optimal parameter before storage or transmission.

\subsection{Moments of Wavelets}

In orthonormal wavelet theory scaling functions $\phi$ are considered with the additional property that their integer translates $\{\phi(x-k)\}_{k \in \mathbb{Z}}$ are orthonormal in $L^{2}(\mathbb{R})$. A wavelet function $\psi$ is associated with $\phi$ via

$$
\psi(x)=\sum_{k=0}^{N}(-1)^{k} h_{N-k} \phi(2 x-k) .
$$

We denote by

$$
N_{n}=\left\langle x^{n}, \psi(x)\right\rangle=\int x^{n} \psi(x) d x
$$

the $n$th continuous moment of the wavelet.

In the sequel we discuss the relation between moments of orthonormal scaling functions $\phi$ and associated wavelets $\psi$. Gopinath \& Burrus [17] and Sweldens \& Piessens [28] established a relation between the first two moments of orthonormal scaling functions and wavelets. This result is generalized to an arbitrary number of moments in Theorem 8.

Theorem 5. [17, 28] If $N_{0}=N_{1}=0$, then

$$
M_{2}=M_{1}^{2}
$$

To establish higher order moment identities we make use of the following two lemmas:

Lemma 6. Let the first $p$ moments of $\psi$ vanish, that is

$$
N_{j}=0, \quad \text { for } j=0, \ldots, p-1 .
$$

Then

$$
\sum_{k}(x-k)^{n} \phi(x-k)=M_{n}, \quad \text { for } 0 \leq n \leq p-1 .
$$

For a proof of this result we refer to Sweldens \& Piessens [28]. 
Lemma 7. Let the first $p$ moments of $\psi$ vanish. Then

$$
\sum_{k} k^{n} \phi(x-k)=\sum_{i=0}^{n}\left(\begin{array}{c}
n \\
i
\end{array}\right)(-1)^{i} x^{n-i} M_{i}, \quad \text { for } 0 \leq n \leq p-1 .
$$

Proof. The proof is done by induction. For $p=1$ the assertion follows from the previous Lemma. Suppose that the assertion is true for $p$ and we assume that $N_{j}=0$ for $j=0, \ldots, p$. We have to show that

$$
\sum_{k} k^{p} \phi(x-k)=\sum_{i=0}^{p}\left(\begin{array}{c}
p \\
i
\end{array}\right)(-1)^{i} x^{p-i} M_{i}
$$

Again from the previous Lemma we know that

$$
\sum_{k}(x-k)^{p} \phi(x-k)=M_{p}
$$

Expanding $(x-k)^{p}$ yields

$$
\sum_{j=0}^{p-1}\left(\begin{array}{l}
p \\
j
\end{array}\right)(-1)^{j} x^{p-j} \sum_{k} k^{j} \phi(x-k)+(-1)^{p} \sum_{k} k^{p} \phi(x-k)=M_{p} .
$$

From this equation and the induction hypothesis it follows that

$$
(-1)^{p+1} \sum_{k} k^{p} \phi(x-k)+M_{p}=\sum_{j=0}^{p-1}\left(\begin{array}{l}
p \\
j
\end{array}\right)(-1)^{j} x^{p-j} \sum_{i=0}^{j}\left(\begin{array}{l}
j \\
i
\end{array}\right)(-1)^{i} x^{j-i} M_{i} .
$$

Interchanging the order of summation and using the identities

$$
\left(\begin{array}{l}
p \\
j
\end{array}\right)\left(\begin{array}{l}
j \\
i
\end{array}\right)=\left(\begin{array}{l}
p \\
i
\end{array}\right)\left(\begin{array}{l}
p-i \\
j-i
\end{array}\right)
$$

and

$$
\sum_{j=i}^{p-1}(-1)^{j}\left(\begin{array}{c}
p-i \\
j-i
\end{array}\right)=(-1)^{p+1}
$$

in (3.21) shows that

$$
\begin{aligned}
(-1)^{p+1} \sum_{k} k^{p} \phi(x-k)+M_{p} & =\sum_{i=0}^{p-1}(-1)^{i} x^{p-i} M_{i} \sum_{j=i}^{p-1}\left(\begin{array}{c}
p \\
j
\end{array}\right)\left(\begin{array}{c}
j \\
i
\end{array}\right)(-1)^{j} \\
& =\sum_{i=0}^{p-1}\left(\begin{array}{c}
p \\
i
\end{array}\right)(-1)^{i} x^{p-i} M_{i}\left(\sum_{j=i}^{p-1}(-1)^{j}\left(\begin{array}{c}
p-i \\
j-i
\end{array}\right)\right) \\
& =(-1)^{p+1} \sum_{i=0}^{p-1}\left(\begin{array}{c}
p \\
i
\end{array}\right)(-1)^{i} x^{p-i} M_{i} .
\end{aligned}
$$

This shows the assertion. 
Using the last lemma we are able to prove a relation between higher order continuous moments of orthonormal scaling functions. This result generalizes Theorem 5.

Theorem 8. Let $\phi \in L^{2}(\mathbb{R})$ be a scaling function with the additional property that its integer translates $\{\phi(x-k)\}_{k \in \mathbb{Z}}$ are orthonormal. Let $p \in \mathbb{N}$ be odd and let the first $p+1$ moments of the associated wavelet $\psi$ vanish. Then

$$
M_{p+1}=\sum_{i=1}^{p}(-1)^{i+1}\left(\begin{array}{c}
p \\
i
\end{array}\right) M_{i} M_{p-i+1} .
$$

Proof. Let

$$
s_{k}=\langle x, \phi(x) \phi(x-k)\rangle, \quad \text { for } k \in \mathbb{Z} .
$$

Since $\phi(x)$ and $\phi(x-k)$ are orthogonal it follows that

$$
s_{-k}=\langle x, \phi(x) \phi(x+k)\rangle=\langle x-k, \phi(x-k) \phi(x)\rangle=s_{k}, \quad \text { for } k \in \mathbb{Z} .
$$

Therefore we get using the assumption that $p$ is odd

$$
0=\sum_{k} k^{p} s_{k}=\left\langle x, \phi(x) \sum_{k} k^{p} \phi(x-k)\right\rangle .
$$

This identity together with (3.20) shows that

$$
0=\sum_{i=0}^{p}\left(\begin{array}{c}
p \\
i
\end{array}\right)(-1)^{i} M_{i}\left\langle x, x^{p-i} \phi(x)\right\rangle=\sum_{i=0}^{p}\left(\begin{array}{c}
p \\
i
\end{array}\right)(-1)^{i} M_{i} M_{p-i+1}
$$

and the proposition follows.

Remark 9. The above theorem reveals that the even moments of an orthonormal scaling function are completely determined by the odd up to the number of vanishing moments of the associated wavelet. We exemplarily give the equations for the even moments using (3.22) for $p=1,3,5$ :

$$
\begin{aligned}
& M_{2}=M_{1}^{2} \\
& M_{4}=-3 M_{2}^{2}+4 M_{1} M_{3}=-3 M_{1}^{4}+4 M_{1} M_{3} \\
& M_{6}=10 M_{3}^{2}+6 M_{1} M_{5}-15 M_{2} M_{4}=45 M_{1}^{6}-60 M_{1}^{3} M_{3}+6 M_{1} M_{5}+10 M_{3}^{2} .
\end{aligned}
$$

Using the relations between continuous and discrete moments from the previous sections, in particular the polynomials $p_{n}$, we obtain from the above equations the following equations for the even discrete moments. We use this observation for the construction of parametrized families of scaling functions in the following subsection.

$$
\begin{aligned}
& m_{2}=\frac{1}{2} m_{1}^{2} \\
& m_{4}=-\frac{1}{2} m_{1}^{4}+2 m_{1}^{2} m_{2}+2 m_{1} m_{3}-\frac{7}{2} m_{2}^{2}=-\frac{3}{8} m_{1}^{4}+2 m_{1} m_{3} \\
& m_{6}=\frac{45}{32} m_{1}^{6}-\frac{15}{2} m_{1}^{3} m_{3}+3 m_{1} m_{5}+5 m_{3}^{2}
\end{aligned}
$$


From the following lemma we obtain a different formulation of the previous theorem.

Lemma 10. Let $n \in \mathbb{N}$ be odd. Let $x_{1}, \ldots, x_{n+1}$ be variables and $x_{0}=1$. Then

$$
\sum_{i=0}^{n}\left(\begin{array}{l}
n \\
i
\end{array}\right)(-1)^{i} x_{i} x_{n-i}=0
$$

and

$$
\sum_{i=0}^{n+1}\left(\begin{array}{c}
n+1 \\
i
\end{array}\right)(-1)^{i} x_{i} x_{n+1-i}=2 x_{n+1}+2 \sum_{i=1}^{n}\left(\begin{array}{c}
n \\
i
\end{array}\right)(-1)^{i} x_{i} x_{n+1-i}
$$

Proof. Since $n$ is odd the first assertion follows from

$$
\begin{aligned}
\sum_{i=0}^{n}\left(\begin{array}{c}
n \\
i
\end{array}\right)(-1)^{i} x_{i} x_{n-i} & =(-1)^{n} \sum_{i=0}^{n}\left(\begin{array}{c}
n \\
n-i
\end{array}\right)(-1)^{-i} x_{n-i} x_{i} \\
& =-\sum_{i=0}^{n}\left(\begin{array}{c}
n \\
i
\end{array}\right)(-1)^{i} x_{i} x_{n-i} .
\end{aligned}
$$

The second assertion follows from

$$
\begin{aligned}
& \sum_{i=0}^{n+1}\left(\begin{array}{c}
n+1 \\
i
\end{array}\right)(-1)^{i} x_{i} x_{n+1-i} \\
= & x_{0} x_{n+1}+(-1)^{n+1} x_{0} x_{n+1}+\sum_{i=1}^{n}\left(\begin{array}{c}
n+1 \\
i
\end{array}\right)(-1)^{i} x_{i} x_{n+1-i} \\
= & 2 x_{n+1}+\sum_{i=1}^{n}\left(\left(\begin{array}{c}
n \\
i
\end{array}\right)+\left(\begin{array}{c}
n \\
i-1
\end{array}\right)\right)(-1)^{i} x_{i} x_{n+1-i}
\end{aligned}
$$

and

$$
\begin{aligned}
\sum_{i=1}^{n}\left(\begin{array}{c}
n \\
i-1
\end{array}\right)(-1)^{i} x_{i} x_{n+1-i} & =(-1)^{n+1} \sum_{i=1}^{n}\left(\begin{array}{c}
n \\
n-i
\end{array}\right)(-1)^{-i} x_{n+1-i} x_{i} \\
& =\sum_{i=1}^{n}\left(\begin{array}{c}
n \\
i
\end{array}\right)(-1)^{i} x_{n+1-i} x_{i} .
\end{aligned}
$$

Corollary 11. Let the first $p$ moments of $\psi$ vanish. Then

$$
\sum_{i=0}^{n}(-1)^{i}\left(\begin{array}{l}
n \\
i
\end{array}\right) M_{i} M_{n-i}=0, \quad \text { for } 1 \leq n \leq p .
$$

Proof. Let $n$ be odd. In this case (3.25) is trivially satisfied by (3.23). Let $n$ be even and $1 \leq n \leq p$, then (3.25) follows from (3.22) from Theorem 8 and (3.24).

Equation (3.25) is well known to hold if there continuous moments are replaced by discrete moments (see for example Bäni [2, p. 115], where the normalization $m_{0}=1$ is used). 


\subsection{Filter Coefficients Parametrized by Moments}

To construct families of parametrized scaling functions and wavelets we use that the even moments are determined by the odd up to number of vanishing moments (cf. Remark 9). The parametrization is with respect to the first moments of the associated scaling function. We derive formulas for filter coefficients of scaling functions with four, six, and eight filter coefficients and, one, two respectively three vanishing moments using symbolic computation.

To this end we recall the basic polynomial equations for the filter coefficients of a scaling function implied by orthonormality and vanishing moments of the associated wavelet, see for example Daubechies [13] or Strang [26]. The orthonormality of the integer translates of the scaling function imply that number of filter coefficients is even. In the following, it is convenient to number the filter coefficients by

$$
h_{k}, \quad \text { for } 1-N \leq k \leq N .
$$

Note that then the discrete moments $m_{n}$ for the filter coefficients are equal to

$$
m_{n}=\sum_{k=1-N}^{N} h_{k}(k+N-1)^{n} .
$$

Orthonormality of the scaling function, $\int \phi(x) \phi(x-l)=\delta_{0, l}$, can be transformed using the dilation equation (1.1) into

$$
\sum_{k=1-N}^{N} h_{k} h_{k-2 l}=2 \delta_{0, l}, \quad \text { for } l=0, \ldots, N-1,
$$

where $h_{k}=0$, for $k<1-N$ or $k>N$. The condition that the first $p$ moments of the associated wavelet

$$
\psi(x)=\sum_{k=1-N}^{N}(-1)^{k} h_{1-k} \phi(2 x-k)
$$

vanish, that is

$$
N_{j}=\int x^{j} \psi(x) d x=0, \quad \text { for } j=0, \ldots, p-1
$$

is equivalent to

$$
\sum_{k=1-N}^{N}(-1)^{k} h_{1-k} k^{l}=0, \quad \text { for } l=0, \ldots, p-1 .
$$

Equation (3.27) for $l=0$ is redundant and thus omitted.

In the following we present the conditional equations for four and six filter coefficient with one degree of freedom achieved by giving up a vanishing moment condition of the standard orthogonal wavelet setting. For four filter coefficients the conditional system consists of two linear equations, resulting from the normalization $m_{0}=2$ (cf. Lemma 1) and the vanishing moment condition (3.28). Using the first discrete moment 
$m:=m_{1}$ as a parameter gives a third linear constraint on the filter coefficients. Thus we have the following system of equations:

$$
\left.\begin{array}{rl}
h_{-1}+h_{0}+h_{1}+h_{2} & =2 \\
-h_{2}+h_{1}-h_{0}+h_{-1} & =0 \\
h_{0}+2 h_{1}+3 h_{2} & =m
\end{array}\right\} \quad \begin{aligned}
& \text { linear equations } \\
& h_{1} h_{-1}+h_{2} h_{0}=0
\end{aligned} \quad \text { quadratic equation } .
$$

Solving the system of linear equations for $h_{2}$ and substituting the solution into the quadratic equation gives

$$
-2 h_{2}^{2}+h_{2} m-h_{2}-1 / 4 m^{2}+m-3 / 4=0 .
$$

This equation has two possible solutions - each of them gives feasible filter coefficients. Let

$$
w=\sqrt{-5+6 m-m^{2}} \text { and } 1 \leq m \leq 5,
$$

then for $h_{2}=-1 / 4+1 / 4 m-1 / 4 w$ we obtain

$$
\begin{aligned}
h_{-1} & =5 / 4-1 / 4 m-1 / 4 w \\
h_{0} & =5 / 4-1 / 4 m+1 / 4 w \\
h_{1} & =-1 / 4+1 / 4 m+1 / 4 w .
\end{aligned}
$$

For $m=3-\sqrt{3}$ and $m=3+\sqrt{3}$ we obtain the classical Daubechies filters with two vanishing moments [12]. The Haar wavelet corresponds to $m=1$ (for $m=3,5$ we get translated versions). The smoothest scaling function with four filter coefficients with respect to the Hölder regularity is obtained for $m=1.4$, see Daubechies [13, p. 242] and Rioul [22].

For six filter coefficients with at least two vanishing moments the resulting system of equations for the filter coefficients is much more involved. Now, the system consists of linear equations resulting from the normalization $m_{0}=2$ (one equation) and the vanishing moment conditions (3.28) (two equations). Using again the first discrete moment $m:=m_{1}$ as a parameter gives a forth linear constraint on the filter coefficients. In Remark 9 it is shown that $m_{2}=m_{1}^{2} / 2$ if the first two moments of the associated wavelet function vanish. This gives a further linear equation. Two quadratic equations follow from the orthonormality of the scaling function (3.27):

$$
\left.\begin{array}{c}
h_{-2}+h_{-1}+h_{0}+h_{1}+h_{2}+h_{3}=2 \\
h_{3}-h_{2}+h_{1}-h_{0}+h_{-1}-h_{-2}=0 \\
-2 h_{3}+h_{2}-h_{0}+2 h_{-1}-3 h_{-2}=0 \\
h_{-1}+2 h_{0}+3 h_{1}+4 h_{2}+5 h_{3}=m \\
h_{-1}+4 h_{0}+9 h_{1}+16 h_{2}+25 h_{3}=m^{2} / 2
\end{array}\right\} \quad \text { linear equations, }
$$

We have solved this system using Gröbner bases with the computer algebra software 
MAPLE and obtained the parametrized solutions:

$$
\begin{aligned}
h_{-2} & =\frac{21}{16}-\frac{7}{16} m+\frac{1}{32} m^{2}-\frac{1}{32} w \\
h_{-1} & =\frac{25}{16}-\frac{7}{16}+\frac{1}{32} m^{2}+\frac{1}{32} w \\
h_{0} & =-\frac{5}{8}+\frac{5}{8} m-\frac{1}{16} m^{2}+\frac{1}{16} w \\
h_{1} & =-\frac{5}{8}+\frac{5}{8} m-\frac{1}{16} m^{2}-\frac{1}{16} w \\
h_{2} & =\frac{5}{16}-\frac{3}{16} m+\frac{1}{32} m^{2}-\frac{1}{32} w \\
h_{3} & =\frac{1}{16}-\frac{3}{16} m+\frac{1}{32} m^{2}+\frac{1}{32} w,
\end{aligned}
$$

with

$$
w=\sqrt{-260+360 m-136 m^{2}+20 m^{3}-m^{4}} \text { and } 5-\sqrt{15} \leq m \leq 5+\sqrt{15} .
$$

The Daubechies wavelet db3 corresponds to

$$
m=5-\sqrt{5-2 \sqrt{10}} \text { or } m=5-\sqrt{5+2 \sqrt{10}}
$$

coiflets belong to the case $m=4$.

The parametrized solutions for eight filter coefficients with at least three vanishing moments:

$$
\begin{aligned}
h_{-3} & =-\frac{1}{512} \frac{m^{5}-42 m^{4}+684 m^{3}-5416 m^{2}+20840 m-31088+w}{m^{2}-14 m+50} \\
h_{-2} & =-\frac{1}{512} \frac{m^{6}-52 m^{5}+1124 m^{4}-12880 m^{3}+82344 m^{2}-278080 m-m w+6 w+387072}{m^{3}-22 m^{2}+162 m-400} \\
h_{-1} & =\frac{1}{512} \frac{3 m^{5}-110 m^{4}+1508 m^{3}-9432 m^{2}+25016 m-16464+3 w}{m^{2}-14 m+50} \\
h_{0} & =\frac{1}{512} \frac{3 m^{6}-140 m^{5}+2636 m^{4}-25360 m^{3}+129144 m^{2}-317760 m-3 m w+18 w+265216}{m^{3}-22 m^{2}+162 m-400} \\
h_{1} & =-\frac{1}{512} \frac{3 m^{5}-94 m^{4}+1092 m^{3}-5944 m^{2}+15416 m-16464+3 w}{m^{2}-14 m+50} \\
h_{2} & =-\frac{1}{512} \frac{3 m^{6}-124 m^{5}+2028 m^{4}-16688 m^{3}+71416 m^{2}-142784 m-3 w m+18 w+86016}{m^{3}-22 m^{2}+162 m-400} \\
h_{3} & =\frac{1}{512} \frac{m^{5}-26 m^{4}+268 m^{3}-1416 m^{2}+4072 m-5488+w}{m^{2}-14 m+50} \\
h_{4} & =\frac{1}{512} \frac{m^{6}-36 m^{5}+516 m^{4}-3696 m^{3}+13352 m^{2}-20160 m-w m+6 w+3072}{m^{3}-22 m^{2}+162 m-400},
\end{aligned}
$$

with

$$
w=\sqrt{-\left(m^{8}-56 m^{7}+1336 m^{6}-17696 m^{5}+141792 m^{4}-699328 m^{3}+2049600 m^{2}-3186176 m+1891904\right)(m-8)^{2}} .
$$

We recall that with Subsection 2.2 the filter coefficients can also be expressed via the continuous moment $M_{1}$. 


\subsection{Parametrized Wavelets and Compression}

Here we discuss a novel concept of data compression using parametrized scaling functions and wavelets. For compression the coefficients of the expansion of the data with respect to scaled and dilated scaling functions and wavelets are computed. The coefficients of the expanded data are transmitted and decoded afterwards. For data compression with parametrized wavelets the expansion is computed for a series of parameters and the one that yields optimal compression rates is selected. The coefficients and the parameter are transmitted. These data are sufficient for decoding.

In the following we present a numerical example for data compression using parametrized wavelets. As data we use a one-dimensional signal from contact less ultrasound measurements for non destructive evaluation of an Aluminum sheet (see Figure 1 left). Figure 1 right shows the "optimal" scaling function and the according wavelet with 8 filter coefficients for approximating the data.
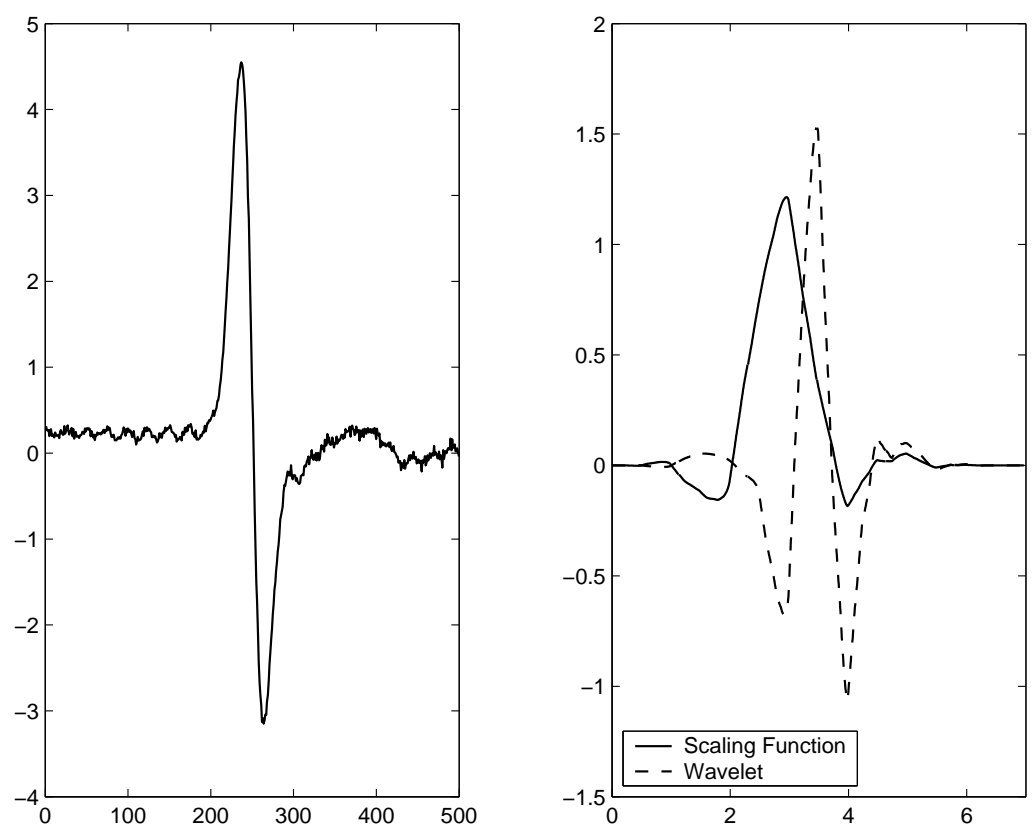

Figure 1: Signal and "optimal" scaling function and wavelet

In the particular example we have only used the coefficients of the scaling function expansion and have set all wavelet coefficients to zero. This is of course not a realistic way of data compression, but more sophisticated approaches can be dealt with analogously.

The error for the decoded data is shown in Figure 2. We also show the result using the db4 (Daubechies 4) scaling function. In comparison the squared error (SE) is about three times as high for $\mathrm{db} 4$ and the maximal error (ME) is about double. The related software is available on request from the first named author. 

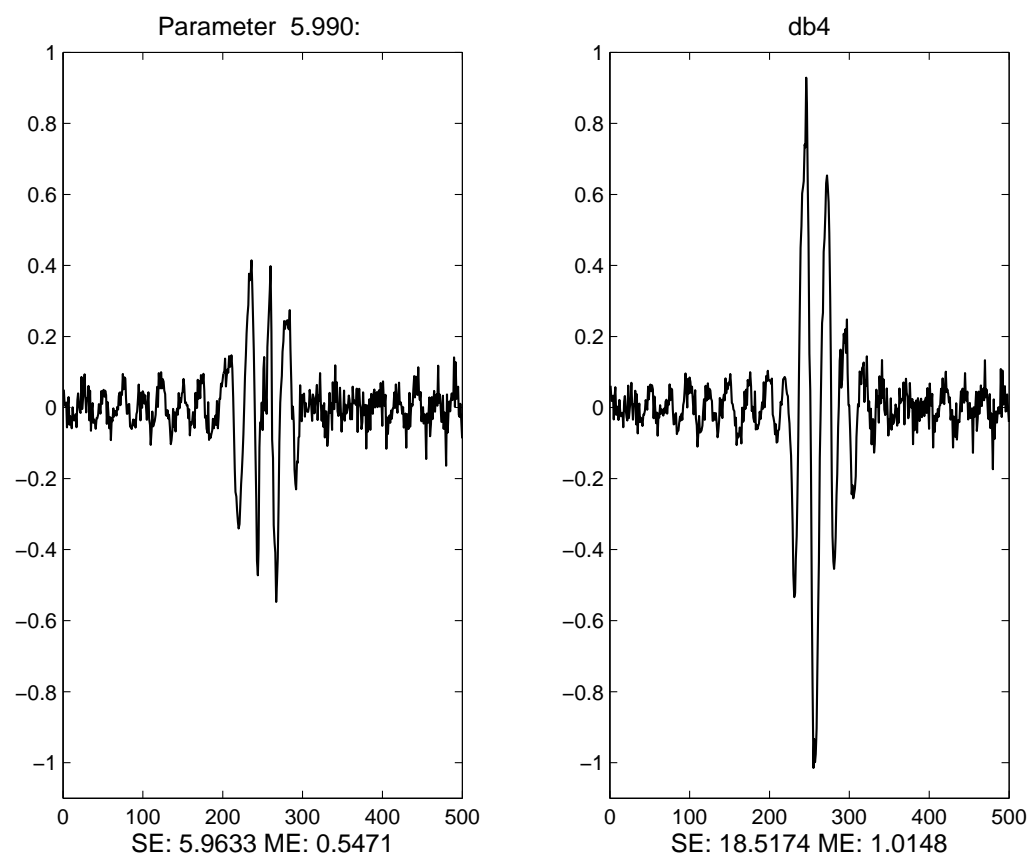

Figure 2: Error for the decoded data

\section{Appendix: Bell Polynomials and Partitions}

Bell polynomials have been introduced by E. T. Bell [3]. Since then Bell polynomials have become a fundamental tool in combinatorics. One classical applications of Bell polynomials is the compact representation of higher order derivatives of composite functions known as the Formula of Faa di Bruno (see Roman [23]). References on Bell polynomials are Comtet [10], Riordan [21], and Roman [24]. For some recent applications of Bell polynomials we refer to Cassisaricci [6] and Collins [9].

Definition 12. Let $n, k \in \mathbb{N}$. The (exponential) partial Bell polynomials are defined by

$$
\begin{aligned}
B_{n, k} & :=B_{n, k}\left(x_{1}, \ldots, x_{n-k+1}\right) \\
& =\frac{1}{k !} \sum_{\substack{i_{1}+\cdots+i_{k}=n \\
i_{j}>0}}\left(\begin{array}{c}
n \\
i_{1}, \ldots, i_{k}
\end{array}\right) x_{i_{1}} \cdots x_{i_{k}} \\
& =\sum_{\substack{k_{1}+\cdots+k_{n}=k \\
k_{1}+2 k_{2}+\cdots+n k_{n}=n \\
k_{i} \geq 0}} \frac{n !}{k_{1} ! k_{2} ! \cdots k_{n} !}\left(\frac{x_{1}}{1 !}\right)^{k_{1}}\left(\frac{x_{2}}{2 !}\right)^{k_{2}} \cdots\left(\frac{x_{n}}{n !}\right)^{k_{n}} .
\end{aligned}
$$

This identity in particular shows that $B_{n, k} \in \mathbb{Z}\left[x_{1}, \ldots, x_{n-k+1}\right]$. We set $B_{n, 0}=0$ and 
$B_{0,0}=1$. The (exponential) Bell polynomials are defined by

$$
Y_{n}=\sum_{k=0}^{n} B_{n, k} \in \mathbb{Z}\left[x_{1}, \ldots, x_{n}\right], \quad \text { for } n \in \mathbb{N}_{0} .
$$

The first five Bell polynomials are:

$$
\begin{aligned}
& Y_{0}=1 \\
& Y_{1}=x_{1} \\
& Y_{2}=x_{1}^{2}+x_{2} \\
& Y_{3}=x_{1}^{3}+3 x_{1} x_{2}+x_{3} \\
& Y_{4}=x_{1}^{4}+6 x_{1}^{2} x_{2}+3 x_{2}^{2}+4 x_{1} x_{3}+x_{4} .
\end{aligned}
$$

They satisfy the recurrence relation

$$
Y_{n+1}\left(x_{1}, \ldots, x_{n+1}\right)=\sum_{i=0}^{n}\left(\begin{array}{c}
n \\
i
\end{array}\right) Y_{n-i}\left(x_{1}, \ldots, x_{n-i}\right) x_{i+1},
$$

see for example Riordan [21, p. 36].

Definition 13. A partition of $n \in \mathbb{N}$ is a nonincreasing sequence of positive integers, denoted by $\left(\lambda_{1}, \ldots, \lambda_{k}\right)$, whose sum is $n$. Each $\lambda_{i}$ is called a part of the partition. $A$ composition of $n \in \mathbb{N}$ is a sequence of positive integers whose sum is $n$.

Example 14. There are five partitions of 4 :

$$
(4),(31),(22),(211),(1111)
$$

and eight compositions:

$$
\text { (4), (31), (13), (22), (211), (121), (112), (1111) . }
$$

A partitions of $n$ in $k$ parts is usually denoted by

$$
1^{k_{1}} 2^{k_{2}} \cdots n^{k_{n}}, \quad \text { with } k_{1}+2 k_{2}+\cdots+n k_{n}=n,
$$

where $k_{i} \in \mathbb{N}_{0}$ is the number of parts equal to $i$ and $k=k_{1}+\cdots+k_{n}$. For instance

$$
(211)=1^{2} 2 .
$$

Using Definition 13 we see that the sums in (4.30), (4.31) are sums over all compositions, partitions, respectively, of $n$ in $k$ parts.

The number of partitions of $n$ is denoted by $p(n)$. Equation (4.31) together with (4.33) show that the number of monomials in the Bell polynomials $Y_{n}$ is the number of partitions of $n$, which increases rapidly with $n$. For example $p(7)=15$ and $p(33)=$ 10143. For further background on partitions we refer to Andrews [1]. 


\section{Acknowledgement}

This work has been supported by the FWF (Fonds zur Förderung der wissenschaftlichen Forschung), grant Y-123 INF-N12.

The authors are grateful to Dr. Peter Burgholzer for providing the ultrasound measurement data Figure 1 left.

\section{References}

1. George E. Andrews. The theory of partitions. Addison-Wesley Publishing Co., Reading, Mass.-London-Amsterdam, 1976. Encyclopedia of Mathematics and its Applications, Vol. 2.

2. Werner Bäni. Wavelets, Eine Einführung für Ingenieure. Oldenbourg Verlag, München, Wien, 2002.

3. Eric Temple Bell. Exponential polynomials. Annals of Mathematics, 35:258-277, 1934.

4. Bruno Buchberger. An Algorithm for Finding the Bases Elements of the Residue Class Ring Modulo a Zero Dimensional Polynomial Ideal (German). PhD thesis, Univ. of Innsbruck, 1965.

5. Bruno Buchberger. Ein algorithmisches Kriterium für die Lösbarkeit eines algebraischen Gleichungssystems. Aequationes Math., 4:374-383, 1970.

6. Caterina Cassisa and Paolo E. Ricci. Orthogonal invariants and the Bell polynomials. Rend. Mat. Appl. (7), 20:293-303, 2000. Dedicated to the memory of Gaetano Fichera (Italian).

7. Alfred S. Cavaretta, Wolfgang Dahmen, and Charles A. Micchelli. Stationary subdivision. Mem. Amer. Math. Soc., 93(453):vi+186, 1991.

8. Frédéric Chyzak, Peter Paule, Otmar Scherzer, Armin Schoisswohl, and Burkhard Zimmermann. The construction of orthonormal wavelets using symbolic methods and a matrix analytical approach for wavelets on the interval. Experimental Mathematics, 10(1):67-86, 2001.

9. C. B. Collins. The role of Bell polynomials in integration. J. Comput. Appl. Math., 131(1-2):195-222, 2001.

10. Louis Comtet. Advanced combinatorics. D. Reidel Publishing Co., Dordrecht, enlarged edition, 1974. The art of finite and infinite expansions.

11. David Cox, John Little, and Donal O'Shea. Ideals, varieties, and algorithms. Undergraduate Texts in Mathematics. Springer-Verlag, New York, second edition, 1997. An introduction to computational algebraic geometry and commutative algebra. 
12. Ingrid Daubechies. Orthonormal bases of compactly supported wavelets. Comm. Pure Appl. Math., 41(7):909-996, 1988.

13. Ingrid Daubechies. Ten lectures on wavelets. Society for Industrial and Applied Mathematics (SIAM), Philadelphia, PA, 1992.

14. Ingrid Daubechies. Orthonormal bases of compactly supported wavelets. II. Variations on a theme. SIAM J. Math. Anal., 24(2):499-519, 1993.

15. Ingrid Daubechies and Jeffrey C. Lagarias. Two-scale difference equations. I. Existence and global regularity of solutions. SIAM J. Math. Anal., 22(5):1388-1410, 1991.

16. Ingrid Daubechies and Jeffrey C. Lagarias. Two-scale difference equations. II. Local regularity, infinite products of matrices and fractals. SIAM J. Math. Anal., 23(4):1031-1079, 1992.

17. Ramesh A. Gopinath and C. Sidney Burrus. On the moments of the scaling function $\psi_{0}$. In Proc. of the IEEE ISCAS, volume 2, pages 963-966, San Diego, CA, 1992.

18. Jérôme Lebrun and Ivan W. Selesnick. Gröbner bases and wavelet design. Journal of Symbolic Computation, 37(2):227-259, February 2004.

19. Jérôme Lebrun and Martin Vetterli. High-order balanced multiwavelets: theory, factorization, and design. IEEE Trans. Signal Process., 49(9):1918-1930, 2001.

20. Stéphane Mallat. A wavelet tour of signal processing. Academic Press Inc., San Diego, CA, 1998.

21. John Riordan. An introduction to combinatorial analysis. Wiley Publications in Mathematical Statistics. John Wiley \& Sons Inc., New York, 1958.

22. Olivier Rioul. Simple regularity criteria for subdivision schemes. SIAM J. Math. Anal., 23(6):1544-1576, 1992.

23. Steven Roman. The formula of Faà di Bruno. Amer. Math. Monthly, 87(10):805809, 1980.

24. Steven Roman. The umbral calculus. Academic Press Inc., New York, 1984.

25. Ivan W. Selesnick and C. Sidney Burrus. Maximally flat low-pass FIR filters with reduced delay. IEEE Transactions on Circuits and Systems II: Analog and Digital Signal Processing, 45(1):53-68, 1998.

26. Gilbert Strang. Wavelets and dilation equations: a brief introduction. SIAM Rev., 31(4):614-627, 1989.

27. Gilbert Strang and Truong Nguyen. Wavelets and filter banks. WellesleyCambridge Press, Wellesley, MA, 1996. 
28. Wim Sweldens and Robert Piessens. Quadrature formulae and asymptotic error expansions for wavelet approximations of smooth functions. SIAM J. Numer. Anal., 31(4):1240-1264, 1994.

29. Michael Unser and Thierry Blu. Wavelet theory demystified. IEEE Transactions on Signal Processing, 51(2):470-483, February 2003. 